\title{
La organización académica de las universidades públicas: entre círculos y cuerpos
}

\author{
Tirso Suárez-Núñez* \\ Leonor López Canto**
}

Los actores no tienen estrategia; de otros reciben los planes de batalla, todos contradictorios.

B. Latour

\section{Resumen}

Una nueva estrategia de cambio apoyada en los denominados Cuerpos Académicos (CA) se despliega en las universidades públicas mexicanas; sin embargo, sus resultados pueden ser irregulares por el uso de una terminología poco clara. Efectivamente, en la literatura y en el discurso oficial, el concepto de CA ya no es usado como sinónimo de comunidad sometida a examen sociológico, si no como grupo disciplinar concebido como un elemento organizacional al cual se le atribuyen responsabilidades y tareas, pero sin precisar los arreglos estructurales y normativos para que cumplan los propósitos que se le asignan; esto conlleva el riesgo de convertirlos en "círculos de calidad" e incluso en simples fachadas. Frente a este panorama, en este trabajo se identifican las insuficiencias e imprecisiones del discurso, las cuales vistas a la luz de teorías de organización académica permiten formular los escenarios posibles y deseables con la intención de clarificar y encauzar el cambio que se busca.

Palabras clave: organización, administración universitaria, universidades públicas, academia, grupos de trabajo.

\footnotetext{
* Profesor-investigador de la Facultad de Contaduría y Administración de la Universidad Autónoma de Yucatán. Correo electrónico: snunez@tunku.uady.mx

** Profesora-investigadora de la Facultad de Contaduría y Administración de la Universidad Autónoma de Yucatán. Correo electrónico: 1canto@tunku.uady.mx
} 
$\mathrm{L}$ as universidades públicas están siendo presionadas por las autoridades edu cativas para que incluyan dentro de su organización y procedimientos a los denominados Cuerpos Académicos (CA en adelante). La elaboración de planes y programas, así como los proyectos de desarrollo organizacional y los de investigación tienen que ser respaldados por los CA que han sido nominados en el discurso oficial como "la fuerza motriz del desarrollo institucional".

Ante esto, resulta muy importante formular las siguientes interrogantes: ¿qué son, en concreto, los CA?, ¿son una mejor opción frente a las formas de organización actuales?, ¿por qué el interés o el impulso a esta forma de organización colegiada?, ¿se requieren adecuaciones en las formas y procedimientos actuales para aprovechar su potencial?

Hasta ahora estas y otras preguntas en el mismo sentido no parecen tener respuestas claras y precisas de la parte oficial; por ende, la introducción de los CA dentro de la organización universitaria, así como su impulso por parte de sus autoridades, está dando lugar a muy variadas interpretaciones, que a su vez generan avances irregulares en su aprovechamiento y provocan la distorsión de sus supuestos fines, pues - debido a las estructuras prevalecientes en la mayoría de las instituciones de educación superior (IES) - en los hechos se corre el riesgo de que muchos de ellos están funcionando como "círculos de calidad", que son esquemas grupales temporales de las empresas creados para plantear y resolver problemas al margen de la jerarquía, pero sin facultades de realización que los vuelve poco efectivos y proclives a la simulación. Este ensayo aspira a señalar las insuficiencias organizacionales que el discurso propicia para, con base en lo que la teoría recomienda, identificar las causas hipotéticas, anticipar las consecuencias y formular los escenarios posibles y deseables con el propósito de contribuir a esclarecer las interrogantes antes planteadas, así como al cambio y desarrollo institucional de las universidades públicas mexicanas.

\section{De la sociología a la organización del conocimiento}

El antecedente más remoto del término CA se encuentra en los trabajos de Manuel Gil Antón y su grupo (Pérez, Grediaga, Gil, Casillas, De Garay y Pizzonia, 1991; Gil et al., 1994), aunque estos autores abordan el tema con un sentido más sociológico que organizacional, siempre se refieren al CA en singular como sinónimo de comunidad académica, es decir, al colectivo de profesores del sistema de 
La organización académica de las universidades públicas: entre círculos y cuerpos

educación superior en México. Ellos mismos declaran como su interés primordial el estudio del surgimiento de la profesión y del mercado laboral del académico a partir de la década de los setenta, periodo en el cual se registra un mayor crecimiento de la matrícula y número de profesores.

Otro antecedente de los CA se encuentra en el documento fundacional del Programa de Mejoramiento del Profesorado (PROMEP), publicado en 1996, cuyo objetivo es definir el perfil y el entorno de trabajo deseable de los profesores del sistema de educación superior pública de México; asimismo, sentar las bases para realizar la transformación requerida en sus condiciones y, con ello, en la calidad de la educación superior. En nuestro país, los CA aparecen por primera vez cuando se mencionan los atributos deseables en el profesor, entre ellos formación completa (doctorado), experiencia apropiada, distribución equilibrada de su tiempo entre las diversas tareas académicas, cobertura de los cursos por los profesores adecuados — de tiempo completo (TC) y de asignatura — para finalmente incluir como requisito que cada institución cuente con un CA articulado entre sí y vinculado con el exterior.

Similar a lo referido en obras de Gil et al., a lo largo del trabajo señalado se va repitiendo el término $\mathrm{CA}$ como sinónimo del conjunto de profesores adscrito a una dependencia universitaria sin precisar sus caracteres organizacionales o de grupo, su forma de integración, ni mucho menos su articulación con las demás partes de la organización y su posición jerárquica. Por ejemplo, la composición deseable del CA se define en relación con el tamaño del plantel de alumnos, especificando diferentes mezclas de profesores con distinto tiempo de dedicación (tiempo completo, medio tiempo y asignatura) según la naturaleza del programa de estudio.

En otro punto se establece que una dependencia de educación superior (DES) de una institución de educación superior (IES) es la responsable de uno o varios programas de estudio (PE) y comprende un CA bien definido (las cursivas son nuestras); concluye señalando que el CA de una DES se considerará formado por los profesores adscritos a la dependencia que desarrollen su actividad docente de manera regular y los profesores o investigadores de tiempo completo de otra dependencia de la misma IES (PROMEP, 1996: 20).

La concepción del CA como una célula básica de la organización universitaria aparece por primera vez en un artículo con la firma del entonces rector general de 
la Universidad Autónoma Metropolitana (ahora subsecretario de Educación Superior e Investigación Científica de la SEP), quien en una ponencia presentada en el XII Congreso Nacional del Posgrado (Rubio-Oca, 1997) se dedica a describir los rasgos invariantes de los CA y casi al final aclara que en cada departamento de la UAM existen lo que se denominan áreas de investigación, que puede concebirse como un cuerpo académico (las cursivas son nuestras) aunque en su interior puede haber varios cuerpos académicos, siempre y cuando estén trabajando las mismas líneas de investigación.

Además de la poca claridad de la definición anterior, llama la atención que el autor de esta ponencia se refiera con mucha naturalidad al PROMEP y lo describa con orgullo, subrayando que es en sí mismo (el PROMEP) la primera política de estado en educación superior que se establece más allá de los periodos sexenales (Rubio-Oca, 1997).

Por su parte, la Asociación Nacional de Universidades e Instituciones de Educación Superior (ANUIES, 1999), en un documento titulado "La educación superior en el siglo XXI. Líneas estratégicas de desarrollo" se refiere de manera indistinta a los CA en los dos niveles señalados antes, es decir, el sociológico y el organizacional. Incluso reporta que hasta septiembre de 1999 el PROMEP había apoyado a 216 CA que agrupaban a 2861 profesores de TC de 42 universidades públicas.

Una de las definiciones oficiales más recientes de los CA es: "Grupos disciplinares o multidisciplinares de profesores-investigadores que comparten una o varias líneas de investigación (estudio) y un conjunto de objetivos y metas" (Rubio-Oca, 2003); o más recientemente se le ha definido como: "Grupos de profesores de tiempo completo que comparten una o varias líneas de generación o aplicación innovadora del conocimiento (LGAC) (investigación o estudio) en temas disciplinares o multidisciplinares y un conjunto de objetivos y metas académicas. Adicionalmente sus integrantes atienden los programas educativos (PE) en varios niveles para el cumplimiento cabal de las funciones institucionales" (PROMEP, 2004).

Estas definiciones no señalan claramente sobre la ventaja de organizarse en CA, además son tan ambiguas como decir que un CA es un conjunto de académicos. En busca de una mejor precisión se encontró otra definición: "no son parte de un órgano colegiado" y "no son estructuras orgánicas" (Rubio-Oca, 2003). Lo primero se puede interpretar como que no son subdivisiones de consejos académicos existentes, lo cual es lógico; pero lo segundo resulta muy desconcertante. 
A cambio, se insiste en los rasgos invariantes de los CA: a) alta habilitación académica, b) intensa vida colegiada, c) alto compromiso institucional y d) integración de redes, así como en su importancia (Rubio-Oca, 2003) en los términos siguientes:

- Fuerza motriz del desarrollo institucional (planeación).

- Responden por la formación de recursos humanos (licenciatura y posgrado).

- Garantizan el cumplimiento de objetivos institucionales.

- Propician ambientes académicos con gran riqueza intelectual.

- Prestigian a la institución.

Asimismo, se identifican diversos grados de desarrollo de los $\mathrm{CA}$-desde los que están surgiendo y sentando las bases iniciales para su desarrollo futuro, hasta aquellos que ya operan con plena madurez-; sus características son (PROMEP, 2004):

CA en formación:

- Tienen identificados a sus integrantes.

- De sus integrantes al menos la mitad tiene el reconocimiento del perfil de seable.

- Tienen definidas las líneas de generación y/o aplicación del conocimiento.

- Tienen identificados algunos cuerpos académicos afines y de alto nivel de otras instituciones del país o del extranjero con quienes desean establecer relaciones.

CA en consolidación:

- Más de la mitad de sus integrantes tiene la máxima habilitación y cuenta con productos de generación y/o aplicación innovadora del conocimiento.

- Una mayoría de sus integrantes tiene reconocimiento del perfil deseable.

- Participan conjuntamente en líneas de generación o aplicación innovadora del conocimiento bien definidas.

- Más de la mitad de quienes lo integran cuentan con amplia experiencia en docencia y en formación de recursos humanos.

- Colaboran con otros CA. 
CA consolidados:

- Casi la totalidad de sus integrantes tienen la máxima habilitación académica que los capacita para generar y/o aplicar innovadoramente el conocimiento de manera independiente.

- Sus integrantes cuentan con amplia experiencia en docencia y en formación de recursos humanos.

- La casi totalidad cuenta con el reconocimiento de perfil deseable por parte de PROMEP.

- Los integrantes tienen un alto compromiso con la institución.

- Sus integrantes colaboran entre sí y su producción es evidencia de ello.

- Demuestran una intensa actividad académica manifiesta en congresos, seminarios, mesas y talleres de trabajo, etc., de manera regular y frecuente.

- Intensa vida colegiada.

- Sostienen una intensa participación en redes de intercambio académico, con sus pares en el país y en el extranjero, así como con organismos e instituciones nacionales y del extranjero.

En los manuales para la elaboración del Programa Integral de Fortalecimiento Institucional 3.0 de la Secretaría de Educación Pública (SEP, 2003) se recalca la importancia de los $\mathrm{CA}$, reconociéndolos como grupos primarios que conforman una dependencia y que responden por los programas educativos (PE), al establecer:

En este proceso de actualización del PIFI, se requiere hacer un proceso de autoevaluación más profundo y participativo en los tres niveles (institucional, $D E S$ y CA-PE). En el proceso de planeación se evaluarán los PE y los CA de cada una de las DES y, establecerán las acciones para fortalecerlos que, priorizadas e integradas, resultarán en el conjunto de acciones de los PE y CA de cada DES, que serán, junto con los resultados de la autoevaluación, los insumos fundamentales para formular los Programas de Fortalecimiento de cada DES y proyectos asociados. Además se establecerán los compromisos que asume la institución (metas-compromiso) tanto en el ámbito institucional como en el de cada una de sus DES.

De la misma manera, el Conacyt (2003), en su convocatoria de Ciencia Básica 2003-2, incluye a los CA dentro de las modalidades de organización universitaria al establecer apoyos financieros a: 
La organización académica de las universidades

públicas: entre círculos y cuerpos

- iniciativas presentadas por un CA o un grupo de investigación.

- iniciativas de colaboración entre un CA o grupo de investigación consolidados y otro(s) en consolidación.

- iniciativas presentadas por redes de CA y/o grupos de investigación.

El cambio es evidente en la documentación oficial: al referirse en plural a los CA, implícitamente ya se les concibe como los grupos operativos o células básicas de la Universidad, pero no queda claro cómo se integrarán para que sus planteamientos y demandas tengan coherencia y sentido; por ejemplo, no se aclara de dónde tomarán el poder para garantizar el cumplimiento de objetivos institucionales porque si bien en la UAM - debido a su organización departamental- los integrantes de las áreas de investigación eligen al jefe del departamento y sus diferentes órganos colegiados canalizan la participación de los CA hacia la cima, ¿cómo se articularán los CA en aquellas universidades que tienen formas organizacionales que no son precisamente departamentales o que no cuentan con tipos de autoridad colegiada? Una respuesta posible es que los CA constituirán mecanismos de asesoría académica a las autoridades de aquellas instituciones cuya estructura organizacional sigue siendo burocrática, pero sin tener el poder para implementar los cambios que, en materia académica, juzguen pertinentes para el desarrollo de esas instituciones; es decir, funcionarán como lo hacen los círculos de calidad en las organizaciones empresariales.

\section{Opciones de organización y autoridad académica}

Para valorar la importancia y la justificación de incluir a los CA en la estructuración de las universidades resulta obligado partir de Clark (1983), uno de los más reconocidos estudiosos del fenómeno organizacional universitario. De acuerdo con este autor, la diferenciación horizontal en una institución universitaria está basada tradicionalmente en las denominadas disciplinas. Las agrupaciones resultantes de esta forma de división del trabajo son las facultades, escuelas o colegios, encargados de la enseñanza de una profesión - como el derecho o la administración de empresas - o de alguna disciplina básica — como las humanidades o las ciencias naturales. Al interior de cada facultad surgen las denominadas cátedras.

Otra opción para organizar el trabajo son las divisiones, que abarcan toda un área del conocimiento, como biología, ciencias sociales, etc. En su interior surgen los departamentos, que comprenden una determinada especialidad perteneciente a 
una profesión (derecho institucional o medicina interna) o bien están conformados por disciplinas enteras, como física o historia (Clark, 1983).

El tipo de autoridad académica que según Clark se usa en el nivel departamental es la colegial, mientras que en el nivel de cátedra es la autoridad personalista $o$ gremial. La autoridad personalista se apoya en criterios que no se encuentran circunscritos por un reglamento burocrático o por alguna norma colegiada; es el poder discrecional del profesor sobre el alumno, cuyo origen se remonta al poder del maestro sobre el aprendiz en los antiguos gremios, reforzado por la doctrina de la libertad de cátedra y de investigación. En otros términos, en la cátedra se ejerce el poder individual basado en el conocimiento. La autoridad colegiada, por su parte, es el control colectivo ejercido por medio de un cuerpo de pares; es un tipo de gobierno que corresponde a lo que se denomina "comunidad académica"; su fuerza justifica la elección del jefe desde abajo en vez de la designación desde arriba por un oficial superior. Es tan natural la autoridad colegiada en los niveles operativos que se ha convertido en una premisa del sistema de educación superior, presentando un fuerte contraste con las formas tradicionales de gerencia en el mundo de los negocios (Clark, 1983: 66-82; Arechavala y Solís, 1999: 49).

Finalmente para Clark, la cátedra concentra las responsabilidades de la unidad académica en una sola persona: el catedrático, quien supervisa las actividades de un ámbito laboral determinado, apoyado por personal subordinado. Ésta es una forma de organización más individualista que contrasta con el departamento que es más colegiado y que distribuye las responsabilidades y el poder entre varios profesores de rango superior semejante; al mismo tiempo admite mayor participación de los profesores asistentes y asociados.

Los sistemas de autoridad basados en la cátedra son fácilmente reconocibles en Europa, pero en México no son tan nítidos; quizás prevalecieron cuando la universidad estaba formada por una mayoría de profesores de asignatura. En la actualidad, se hace más evidente la presencia de la autoridad burocrática en las universidades mexicanas, la cual surge cuando el cuerpo de profesores es encabezado por un director o equivalente nombrado por la autoridad superior: el rector (Arechavala y Solís, 1999: 48-49). En resumen, son dos las fuentes de poder inherentes a la organización por facultades: en la cátedra es el expertismo, mientras que en la burocracia es la autoridad administrativa. 
La organización académica de las universidades públicas: entre círculos y cuerpos

Una visión más actual es la proporcionada por Montaño (2001) cuando se refiere a las conformaciones organizacionales universitarias, las cuales se derivan de la consideración de que los fines y los medios organizacionales sean únicos y claros o múltiples y ambiguos; con base en el manejo de las opciones que lo anterior representa se desarrollaron cuatro conformaciones ideales (modelos) de organización universitaria: la burocrática, la política, la colegiada y la anárquico-organizada, cuyos rasgos principales se destacan a continuación:

- La burocrática, al contar con fines y medios únicos y claros, se considera la más fácilmente administrable pero con riesgos altos de solidificación estructural, se asocia con universidades cuya labor tradicional y mayoritaria es la docencia.

- La colegiada, surge cuando se considera que los medios son múltiples y ambiguos, lo que implica una mayor participación de los especialistas que interiorizan con mayor facilidad las labores académicas: la labor colectiva destaca frente a la labor individual de la configuración anterior y en ella puede anidar y vincularse más fácilmente la investigación y la docencia.

- La política, enfatiza los fines reconociendo la multiplicidad de los actores, quienes proponen diversas modalidades interpretativas a nivel institucional. La inscripción social de la universidad, su propia legitimidad, es cuestionada por diversos grupos y cualquier solución siempre va a generar insatisfacción y conflicto, en consecuencia la organización se vuelve inestable y sus energías son invertidas en la conciliación y negociación.

- La anarquía organizacional, es una configuración radical ya que asume que tanto fines como medios son múltiples y ambiguos, representa la forma más alejada de la burocracia y en ella se conjuntan aspectos del modelo colegiado y del político. Supuestamente esta configuración es la más adecuada para la investigación de frontera con pequeños grupos de trabajo relativamente autónomos, hábiles en la consecución de fondos económicos para la realización de sus proyectos y no se somete fácilmente a los requerimientos administrativos como planeación, presupuestación, evaluación, etc. Adicionalmente en la mayoría de los casos, existe poco interés en la docencia, a la cual se le mira como una actividad menor. 
De éstas, la burocrática y la colegiada son las más viables como formas de organización universitaria. A continuación se presentan algunas consecuencias derivadas de la adopción de cualquiera de las formas (Montaño, 2001):

En el caso de la conformación burocrática se encuentra lo siguiente:

- Énfasis en el bienestar de los estudiantes, más que en el desarrollo de la disciplina.

- Resultados educativos establecidos con precisión y criterios de evaluación correspondientes: uso intensivo de índices.

- Énfasis en los estudios de licenciatura orientados hacia la práctica profesional.

- Uso de materiales didácticos novedosos, tecnología educativa de punta.

- Profesores preferentemente de asignatura.

- Reducido gasto en investigación.

- Producción de materiales didácticos propios.

La conformación colegiada, en contraste, genera consecuencias como:

- Autonomía de trabajo: libertad académica.

- Participación académica en comités. Desconocimiento de la autoridad formal.

- Énfasis en la investigación. Desinterés por los aspectos financieros.

- El aprendizaje se realiza por imitación y no por transmisión. Trabajo aislado, con algunos discípulos.

- Selección de directivos por méritos académicos. La disciplina es el elemento integrador central.

- Formación integral ajena a los requerimientos laborales.

- Beneficios a la humanidad, no individuales.

El estudio de las culturas académicas y la posibilidad de cambiarlas es otra forma de vislumbrar el fenómeno; Ian McNay, citado por Taylor (1999), tipifica cuatro culturas académicas identificadas a partir de dos dimensiones: a) la definición de políticas y b) el control de su instrumentación. Cada una de estas culturas representa un continuo en un eje que va de "flojo" a "firme"; ambos ejes se intersectan originando cuatro cuadrantes, cada uno de los cuales representa una cultura diferente. De esta manera, en la figura 1 se muestran las cuatro siguientes culturas académicas universitarias: 


\section{FIGURA 1}

\section{Cuatro Culturas Universitarias}

Control de instrumentación

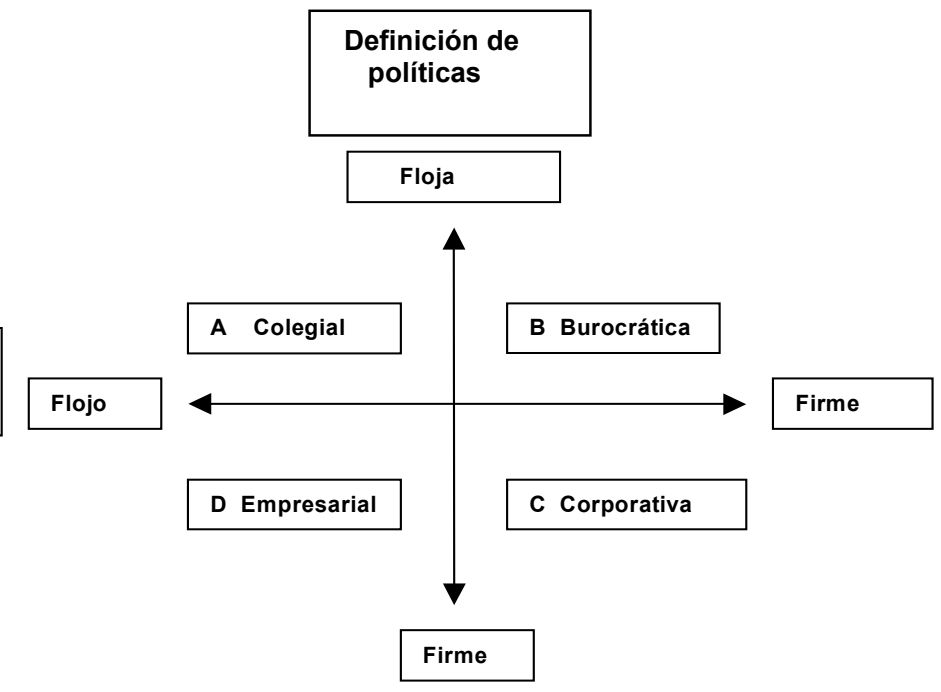

- Tipo A o colegial: floja definición de políticas y flojo control de su instrumentación. Es la tradicional cultura colegial que enfatiza la libertad académica y la autonomía con el personal organizado en departamentos por disciplina, donde la toma de decisiones es consensual y a menudo ejercida mediante comités y con énfasis en evaluaciones por pares.

- Tipo B o burocrática: floja definición de las políticas y firme control de su instrumentación. Es una dirección basada en el managerialism con un fuerte enfoque en el control, consistencia y predictibilidad. El poder radica en quien tiene la facultad de auditar; por ello, hay una fuerte influencia de reguladores externos, asociaciones de profesionales y similares.

- Tipo C o corporativa: firme definición de la política y de su instrumentación. Tanto el liderazgo como la dirección son importantes atributos de cultura. Se brinda una alta prioridad y detalle a la planeación y programación financiera; posteriormente, la evaluación se convierte en un aspecto crucial porque todo el proceso está a cargo de directivos cuyo poder y autoridad permanece centralizado en niveles superiores.

- Tipo D o empresarial: firme control en la definición de la política y flojo control de su instrumentación. El énfasis recae en el liderazgo que define la misión y estable- 
Tirso Suárez-Núñez

ce la dirección; pero los detalles de la instrumentación se deja a los operadores académicos, quienes necesitan libertad para responder oportunamente a los desafíos locales. El poder en esta cultura reside en los emprendedores y en los "clientes".

Es cierto que las universidades no son monoculturales, pues en ellas se presentan, en diferente proporción, partes de cada una de las cuatro culturas antes mencionadas. Sin embargo, es un hecho que actualmente está variando el balance de la proporción de cada una; por ejemplo, en Australia se reporta un cambio - con una tendencia a disminuir la cultura colegial y aumentar la corporativa - como resultado de una reestructuración para volver más competitivas a las universidades en ciertos nichos y aumentar la productividad de sus miembros. No obstante, estos cambios no son los más recomendables en contextos donde se cuenta con una importante cultura y se requiere una orientación externa; en esos casos la cultura más recomendada es la empresarial. Adicionalmente, los ajustes necesarios sólo implican encauzar las fuerzas culturales y no disolverlas, lo que se logra introduciendo la lealtad institucional junto a la de la disciplina, algo equivalente a la doble ciudadanía (Taylor, 1999: 76-78).

En el caso mexicano, el impulso a los CA parece ser un medio para transitar a la cultura colegial, pero al no hacerse las modificaciones estructurales para no perder el control de la definición de la política se sientan las bases para que surjan contradicciones que pueden generar tensiones y simulaciones.

Por otra parte, investigaciones más recientes de Clark (1995) revelan que las naciones con mayor avance en educación superior en el mundo occidental (Francia, Estados Unidos, Alemania, Inglaterra y Japón) partiendo de ciertos arreglos organizacionales en sus sistemas universitarios buscan favorecer de manera vigorosa la investigación como base para la enseñanza y estudio. En el nivel de las unidades básicas, es decir de facultad o departamento, dichas naciones buscan vincular de manera estrecha y efectiva la enseñanza y el aprendizaje con la investigación, lo cual incorpora y pone en movimiento dos formas de conocimiento: el tangible (o explícito) y el tácito; cada uno requiere, para su difusión y promoción, un tipo particular de vehículo.

Cuando se agrega a la distinción de conocimiento tácito-tangible la idea de unidades básicas, que sirven de vehículos transportadores, surgen como elementos cla- 
La organización académica de las universidades

públicas: entre círculos y cuerpos

ves los grupos de investigación y el departamento, en cuyo seno los estudiantes son sometidos a un flujo de conocimientos de ambas clases. Los grupos de investigación transmiten el conocimiento tácito; los grupos de docencia, que conforman el departamento, propagan el conocimiento explícito, además, controlan la admisión, el progreso de los estudiantes y otorgan los grados; de esta manera los estudiantes, de principio a fin, están bajo el control del departamento y participando en grupos de investigación.

Así, más de lo que generalmente se admite, el departamento es en principio una estructura de enseñanza en cuyo interior se involucran los grupos de investigación, donde de manera notable sus miembros simultáneamente forman parte de la estructura de enseñanza. La unidad organizacional básica resultante tiene así dos lados interconectados: una estructura de grupos de enseñanza y otra de grupos de investigación. La fusión cotidiana de esos grupos es el "nexo institucional", el medio organizacional moderno para mezclar de manera sistemática investigación, docencia y estudio (Clark, 1995).

Ningún grupo por sí solo es capaz de transmitir el conocimiento tangible y el tácito, se requiere siempre de ambos. De la misma manera, resulta ocioso discutir si el departamento o el grupo de investigación es la unidad esencial, puesto que los dos son necesarios porque la ausencia de uno de ellos constituye una gran deficiencia estructural.

En conclusión, según Clark (1995), en la mayor parte de los países se han convencido de que la participación en investigación es la mejor forma de estudiar y enseñar; por lo tanto, fortalecer el nexo investigación-enseñanza-estudio en el nivel operativo de las universidades es muy importante; para ello se requieren los grupos de investigación. En la moderna universidad, la fusión del departamento y el grupo de investigación equivale al matrimonio de la educación superior y la ciencia.

Parece claro que conforme a Clark y Montaño, los denominados CA son grupos de investigación-docencia, células básicas de los departamentos cuya autoridad es colegiada; por lo tanto, la adopción de cuerpos académicos como esquema organizacional en instituciones basadas en facultades y cátedras requeriría adoptar la forma departamental. Pero, sobre todo, sería necesario transitar a estilos de autoridad colegiados y participativos, lo cual en muchas universidades puede representar un cambio radical, tanto por lo que se refiere al aspecto estructural como por el cambio de actitud y estilos de trabajo por parte de los académicos. 
En términos de Montaño, impulsar los CA en las universidades implica cambiar las actuales conformaciones burocráticas o políticas — que distinguen a muchas de ellas - hacia formas colegiadas o anárquicas; este impulso de cambio es para reconocer que los medios para lograr los fines de las universidades son múltiples y ambiguos; es decir, las autoridades educativas, al enfatizar que la investigación es un fin obligado para las universidades, reconocen la diversidad de medios que la tarea de la investigación conlleva, lo que a su vez, las conduce a impulsar, aunque no abiertamente, la adopción de las formas colegiadas y/o anárquicas. Frente a esto, cabe preguntarse ¿por qué no decirlo claro?, ¿por qué el silencio?

\section{Fuerte es el silencio}

Según Ibarra (2001), ante las transformaciones organizacionales que las nuevas formas de operación demandan a las universidades públicas sobresale el silencio de las autoridades. Por una parte, los intereses en juego son una invitación a callar, a graduar, incluso a posponer las modificaciones; por otra, se argumenta que los cambios necesarios son mínimos porque —según los involucrados—son de forma.

Este silencio se explica por la ausencia de estudios sobre los aspectos organizacionales de la universidad, que a su vez se deriva del hecho de que la mayor parte de los trabajos sobre el tema han sido realizados por autores muy cercanos a los espacios de poder de la propia universidad e incluso más allá. Esto queda en evidencia al observarse el flujo continuo de estos autores entre la academia y la política universitaria, que produce un vínculo entre los estudios de la universidad y la conducción de la misma con el consiguiente desdibujamiento de la frontera entre ambas (Ibarra, 2001).

La ausencia organizacional, sigue diciendo Ibarra, favorece a ciertas fuerzas institucionales que no se manifiestan al respecto, pero sí desvían o distraen - mediante sus estrategias y campos de acción - posibles actos de resistencia que pudieran modificar el estado de las relaciones. De esta manera, el gobierno de individuos y poblaciones se efectúa casi siempre con una visión vertical y centralizada, mientras tanto se estimulan debates sobre los grandes problemas y se operan las formas de gobierno excluyentes.

Pero la importancia de la organización, como estructura, acción y proceso, radica en la forma debido a que ningún fin, por válido que sea, tendrá sentido si no se 
cuida la pertinencia de los medios para su consecución. Actualmente, la forma es fondo. Pero ¿cuál es la intención de negarle méritos a la forma? De acuerdo con Ibarra, hay una clara racionalidad e interés del sistema como la causa principal para prolongar un estado de cosas que le conviene.

Otras explicaciones del fenómeno dignas de ser exploradas serían:

a) Que la situación que se vive es un resultado de la ambigüedad característico de los sistemas flojamente acoplados, como lo postula Weick (1976).

b) La aplicación de la "tecnología de la tontería" para usar los términos de March (1989), que consiste en actuar sin objetivos previos.

c) La ilusión de la planeación y política de cambio imaginada por los funcionarios desde su bunker, la cual es finamente ilustrada por Porter (2003).

Un dato en apoyo a la explicación de Weick surge del mismo PROMEP cuando al impulsar el establecimiento de redes con los CA consolidados se le cuestionaba ¿dónde están y quiénes son los CA consolidados? Ante la insistencia, este documento dio a conocer públicamente (noviembre de 2003) en su página web la relación de "CA reconocidos" incluyendo el nombre, la institución de adscripción, las líneas de generación y aplicación de conocimiento, sus integrantes y su grado de consolidación. Destacan en esa lista los CA de la UAM —al menos en la sección de ciencias sociales y administrativas - en la cual se reportan como adscritos a esta institución 13 de un total de 23 CA clasificados como "en consolidación" y un único CA consolidado. Es importante señalar, por otra parte, que en la lista de "CA reconocidos" no se encuentren registros de centros educativos de la talla de El Colegio de México o del Centro de Investigación y Docencia Económica (CIDE).

Más aún, un examen detallado de los "CA reconocidos" de la UAM en la sección de Ciencias Sociales y Administrativas del PROMEP, parecía indicar que éstos - por sus líneas de investigación y el número de personas que los integranatraviesan o desdibujan las áreas de investigación que son la base organizacional de esa institución, aumentando con ello la confusión sobre la esencia del CA como núcleo operativo. 
Tirso Suárez-Núñez

Leonor López Canto

\section{Los círculos de calidad y los equipos de trabajo}

Parece necesario hacer una pausa en el examen de las formas de organización universitaria para revisar aspectos muy puntuales de la organización industrial, como los círculos de calidad, y términos de los cuales se ha abusado en extremo en el discurso gerencial, como "trabajo en equipo", en virtud de que ambos parecen ofrecerse como fórmulas avanzadas, incluso para las universidades.

Los círculos de calidad, calidad total y equipos de trabajo son modelos de participación directa en la toma de decisiones en ámbitos operativos; en México son los únicos modelos de cogestión disponibles, pues se trata de una cogestión hacia abajo, hacia los puestos de trabajo y hacia los empleados, a diferencia de la cogestión representativa como la que se desarrolló en Europa, donde los trabajadores formaban comités de fábrica o tenían representantes en los órganos directivos. La participación directa es una doctrina gerencial que significa una nueva valoración de los recursos humanos por el management, por la cual las gerencias han visualizado al personal como el nuevo eje de modernización y racionalización; es una doctrina gerencial y no social, orientada exclusivamente al incremento de la productividad y la calidad.

Específicamente, los círculos de calidad son organizaciones micro a nivel de la producción, que existen en paralelo a la organización regular del trabajo. Su meta es la solución a los problemas de producción y el mejoramiento continuo de la calidad de productos; lo que se conseguirá con la participación activa de los trabajadores. Un círculo de calidad integra un pequeño grupo de 6 a 12 participantes que se reúne una vez por semana y normalmente durante la jornada de trabajo. Bajo la coordinación del moderador se discuten y se intentan resolver problemas del área de trabajo, del producto y de las relaciones humanas; es decir, mejorar el grado de cooperación y eliminar ausentismo. La fórmula que consiste en convertir a los afectados en participantes activos significa la intención, por parte de la gerencia, de optimizar el trabajo y la responsabilidad del trabajador por la vía de la participación directa (Besunsan, De la Garza, Furstemberg \& Terán, 1995: 15, 17).

En contraste, los equipos de trabajo integran la participación activa y directa a los procesos de producción; son una organización de trabajo regular y asumen funciones de un área determinada de la producción — antes reservada a la gerencia media, como el mantenimiento y la programación-; sin embargo, su funcionamiento no es tan armónico como lo divulga el discurso gerencial. 
La organización académica de las universidades

públicas: entre círculos y cuerpos

Siguiendo a March (1994), un equipo es un constructo teórico, una colección de individuos con problemas de incertidumbre, pero en principio sin conflicto de intereses e identidades. Dicho de otra forma, un equipo es un grupo de personas con identidades y preferencias consistentes; esto es, que lo que gana un individuo lo ganan los otros, además de que no hay problemas de repartición de las ganancias, lo que implica que cada acción ejecutada por un individuo es consistente con las acciones ejecutadas por cualquier otro individuo cuya identidad sea evocada por los demás involucrados como perteneciente al grupo.

Por otra parte, aun si en una situación de múltiples actores hay consistencia y, por lo tanto, el conflicto no es un problema, la comunicación y coordinación sí pueden llegar a serlo; por ejemplo, dos individuos con preferencias consistentes fallan en lograr sus mejores resultados porque no se coordinan o si bien tienen identidades consistentes pueden fracasar porque fallan en ajustar sus identidades de manera conjunta. Por lo anterior, los equipos rara vez se encuentran en su forma pura, consecuentemente lo que en las grandes burocracias de empresas, gobierno y organizaciones religiosas se denomina de manera convencional como equipos, se ha justificado en, al menos, tres maneras (March, 1994: 104-105):

- equipos como aproximaciones: algunas situaciones de múltiples actores involucran individuos con preferencias e identidades que son lo suficientemente próximos para ser considerados como consistentes, de manera que denominarlos equipo es una razonable aproximación;

- equipos como simplificaciones: en algunas situaciones de múltiples actores, los individuos se organizan en numerosos grupos como en casi todas las grandes organizaciones. Con el objeto de destacar las consistencias entre los grupos, se ignoran las inconsistencias al interior de cada uno de ellos y éstos son tratados como equipos;

- equipos como contratos: en algunas teorías de decisiones de múltiples actores, las decisiones son consideradas en dos etapas, en la primera las inconsistencias son eliminadas por un proceso de negociación, compensaciones y acuerdos que definen un contrato que liga a las partes. En la segunda etapa los múltiples actores operan como un equipo. 
Con lo anterior se busca destacar que los círculos de calidad y los equipos de trabajo representan alternativas organizacionales que parecen estar muy alejadas de la esencia del trabajo académico, cuya naturaleza genera múltiples identidades e intereses y cuyos atributos culturales particulares se definen y refuerzan por la alimentación del mito, la identificación de símbolos unificadores, la canonización de ejemplares representativos y la formación de corporaciones con sus límites territoriales que son simultáneamente desdibujados y debilitados por un conjunto de presiones opuestas. Asimismo, estos círculos son especies de tribus que delimitan y luchan por un territorio $\mathrm{y}$, por ende, son muy distantes a las organizaciones industriales que son más racionales y fuertemente acopladas (Becher, 2001: 28-55).

Vale la pena señalar en este punto las diferencias organizacionales de la empresa y la universidad (Arechavala y Solís, 1999: 26):

- Los objetivos de las universidades son ambiguos, diversos y en ocasiones más contradictorios que los objetivos de las empresas privadas;

- Las universidades cuentan con tecnologías poco claras para llevar a cabo la docencia, la investigación y la difusión de la cultura, que se basan más en las habilidades profesionales de los académicos que en procedimientos estandarizados de operación, como ocurre generalmente en la empresa privada;

- Las universidades representan una menor especialización de las actividades de trabajo, ya que en la esencia los profesores asistentes, asociados y titulares realizan lo mismo pero muestran una gran diversidad disciplinaria en las formas especificas de llevar a cabo la docencia y la investigación, menores niveles jerárquicos, menor interdependencia entre las unidades organizacionales, menor control sobre la "materia prima" —particularmente en universidades públicas, donde la admisión suele no ser tan selectiva-, menor capacidad para contabilizar resultados de sus actividades y una mucha mayor tradición simbólica en las actividades que realiza.

- Las diferencias entre universidades y empresas privadas son lo suficientemente importantes como para considerar que los sistemas de coordinación y control, es decir la forma de gobierno, no tienen las mismas consecuencias en ambos tipos de organizaciones, particularmente, se puede señalar que difieren en el tipo de liderazgo que ejercen. 
La organización académica de las universidades públicas: entre círculos y cuerpos

De acuerdo con Arechavala y Solís (1999: 50), las ideas optimistas sobre el "trabajo en equipo" se ven cuestionadas cuando se constata que el supuesto implícito en el modelo colegial — es decir, la participación de profesores, alumnos y administrativos en el proceso de toma de decisiones mejora la calidad de éstas - no siempre se cumple. Hay obstáculos recurrentes, como el temor al deterioro de la autoridad y la falta de confianza - y en ocasiones de respeto- entre grupos de profesores y el cuerpo administrativo. Incluso en ocasiones los grupos se politizan y paralizan debido a que, entre intelectuales, las decisiones que conciernen a las cuestiones relacionadas con las prioridades de investigación o las orientaciones teóricas de los cursos crean disentimientos que no se pueden resolver de manera colegiada porque existen limitaciones derivadas de la habilidad típica de los profesores para exponer ideas, que no siempre conducen a la integración de los puntos de vista y frecuentemente enfatizan diferencias en una franca inclinación a la anarquía organizacional.

\section{Conclusión, prescripción y escenarios posibles}

Existe un ambiente de confusión con respecto a qué son los Cuerpos Académicos, propiciado por la poca claridad en la documentación relacionada con el tema emanada de las instancias oficiales del sistema de educación superior en México, como se vio en la revisión del concepto. Como resultado, cada universidad (e incluso al interior de ellas) puede asignar una distinta interpretación a qué son los $\mathrm{CA}$, cómo funcionan y los requerimientos que su operación demanda donde — más que un intento deliberado de dividir o cooptar - sean el error y el aprendizaje experiencial los elementos explicativos.

En general es evidente que el impulso a la adopción de los CA en las universidades públicas mexicanas no parece provenir de una demanda de los propios académicos como un medio para organizarse y dirigir sus esfuerzos hacia modelos colegiados, sino que viene de otro lado; es decir, de las autoridades educativas en el nivel nacional, quienes aparentemente ven la formación de CA como una estrategia para potenciar el desarrollo de las universidades, buscando promover — dado el estado actual de las cosas - la investigación. Introducir un elemento clave del modelo departamental como son los CA, cuya función primordial es vincular la investigación y la docencia, en universidades organizadas por facultades, donde la tradición ha sido la docencia y, por lo tanto, se carece de normas y hábitos propios para la vida colegial, requiere el conocimiento preciso y la aceptación de los académicos de las nuevas formas para asumir este cambio en sus estilos tradi- 
cionales de trabajo, así como su compromiso para participar activamente en la definición y revisión de las políticas institucionales, característica del modelo colegial. Adicionalmente, aparejado a la adopción de estas células operativas, esas mismas autoridades debieran impulsar con decisión y de manera explícita, el tránsito del modelo tradicional prevaleciente hacia la forma departamental.

Pero también la concentración de muchas universidades públicas mexicanas en la enseñanza de disciplinas más profesionales que científicas ha propiciado en ellas la presencia de estructuras de tipo burocrático, lo que dificulta el establecimiento de la forma departamental y la autoridad colegial, las cuales se adecuan de mejor manera a las exigencias de la investigación.

Otro aspecto importante por verificar en estudios posteriores se refiere a que las autoridades federales - debido al desconocimiento de las características particulares y consecuencias específicas de las universidades - pudieran estar obstaculizando involuntariamente la transición hacia el modelo colegiado; es decir, habría que verificar en qué medida las autoridades federales al pretender que los académicos cambien las formas tradicionales con las que han trabajado y enfoquen sus esfuerzos de lo individual a lo colectivo - sin tomar en cuenta la complejidad que está detrás de este proceso y adicionalmente sin crear el ambiente propicioestuvieran con sus acciones y decisiones prolongando artificialmente la cristalización de los esfuerzos en resultados tangibles en el mediano plazo.

Desde luego que ésta no es la única explicación posible. No hay que olvidar que el hecho de transferir la toma de decisiones académicas - hasta ahora casi siempre en manos de la alta administración universitaria - a esas células básicas denominadas Cuerpos Académicos implicaría restarles poder a las primeras, lo que hace muy probable que ellas no estén dispuestas a asumir el nuevo papel que les correspondería: gestoras de los requerimientos, necesidades y decisiones identificados y demandados por los CA. Y esto porque, como ya se dijo, actualmente el nombramiento de la mayor parte de esas autoridades no se realiza tomando realmente en cuenta a la comunidad académica.

En medio de la situación confusa e insuficiente del discurso oficial con relación a los CA en las universidades públicas, se vislumbra como una medida razonable que las autoridades educativas definan el "tipo ideal" de CA y que reconozcan de manera clara y explícita el contexto necesario para su desarrollo, a fin de que cada universidad plantee etapas y programas de adaptación de sus estructuras y de su 
normatividad — que también sean claros y explícitos — para ir de manera ordenada y definida hacia su adopción.

Se sugiere que el tipo ideal de CA tome en cuenta los criterios siguientes:

- Un CA debe ser un núcleo de profesores cuyo interés radique en una disciplina o en una especialidad científica o profesional, cuya agrupación debe dar lugar a un departamento.

- Los CA deben constituir la célula básica de la organización y se les debe reconocer de manera explícita dentro de la estructura formal, condición necesaria para poder planear el desarrollo de sus disciplinas y especialidades.

- Un CA puede tener una o más líneas de generación y aplicación del conocimiento (LGAC).

- Congruentes con sus LGAC, los CA deben promover la integración de proyectos de investigación o programas educativos con otros CA del mismo departamento, división, unidad, institución, incluso de otras instituciones.

- La flexibilidad, movilidad y transdisciplinariedad de los CA debe estar dada por la definición y redefinición de sus LGAC, proyectos de investigación y programas educativos que conformen por sí mismos o con otros CA (Solís, 2001).

La articulación de los criterios anteriores se aprecia en la figura 2 donde, a manera de ejemplo, se muestra:

- Una dependencia integrada por tres CA.

- Cada CA tiene una o más LGAC. Los CA 1 y 3 desarrollan sendas líneas, mientras que el CA 2 tiene dos.

- Los CA comparten la responsabilidad del desarrollo de un solo proyecto de investigación (PI).

- Los CA responden por un programa educativo (PE) y colaboran en la revisión y desarrollo de PE a cargo de los otros CA de la misma dependencia. 


\section{Figura 2}

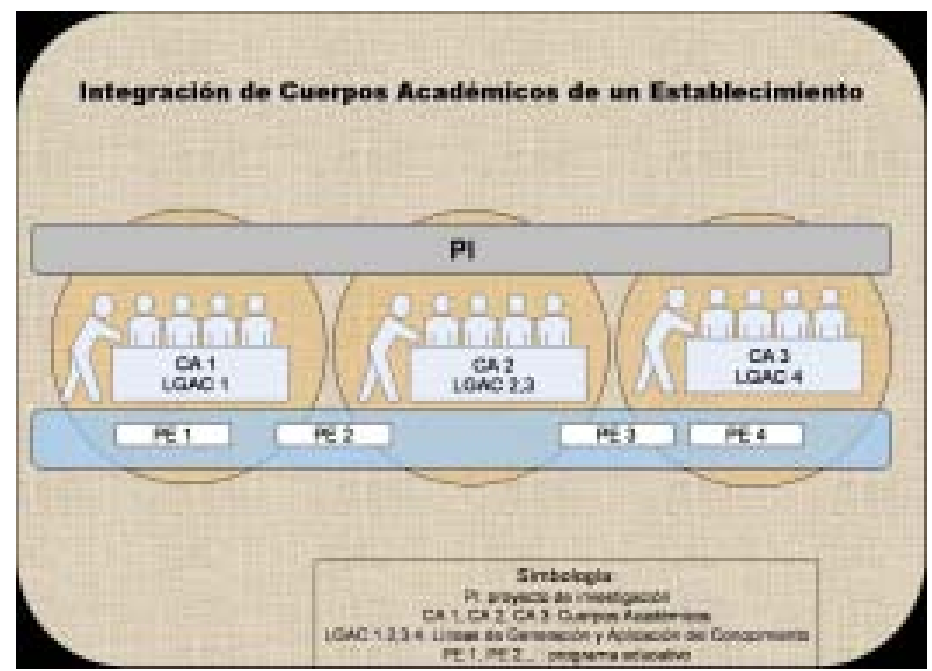

Cabe aclarar que en la figura 2 no aparecen los vínculos que los CA pueden tener con proyectos de investigación o programas educativos de otros CA de otra dependencia e incluso de otra institución.

Asimismo, un CA requiere estar encuadrado en una estructura como la que se muestra en la figura 3, donde se observa que queda establecida una relación directa con el siguiente nivel que es el jefe de departamento; pero esta relación no es jerárquica debido a que el jefe del departamento tiene como función la creación de las condiciones para que los académicos desarrollen su trabajo, apoyándose en las coordinaciones de servicios y de programas educativos. Adicionalmente, son los CA — al constituirse en Consejo Académico — los que dictan el rumbo y la política del departamento. 
Figura 3

Forma departamental

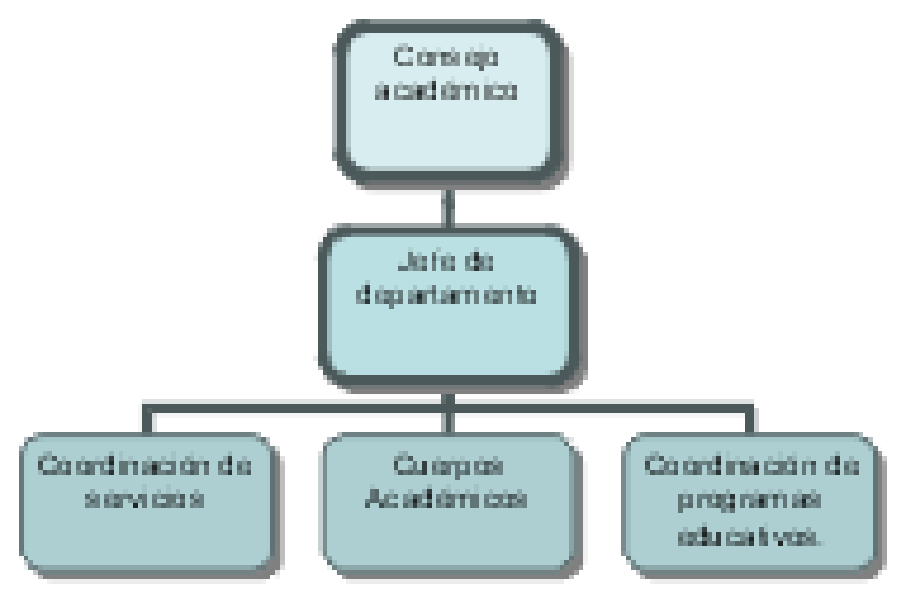

En resumen, la probabilidad de que el escenario deseable sintetizado en la figura 3 prevalezca estará en función del número de universidades que manifiesten claramente su decisión de ir de su estado actual al tipo ideal departamental y programen formalmente la reforma estructural necesaria, evitando quedar por tiempo indefinido en una etapa de transición, que únicamente generaría mayor confusión.

Pero, de no haber una clarificación y ordenación de ideas y conceptos, se vuelven plausibles los siguientes escenarios:

1. Simular que se trabaja con los CA, pero en el fondo la autoridad burocrática prevalece, mientras que los CA permanecen subordinados al Secretario Académico, lo cual desalienta su capacidad autoorganizativa. Es el escenario menos deseado, pero no menos probable (ver figura 4). 
Figura 4

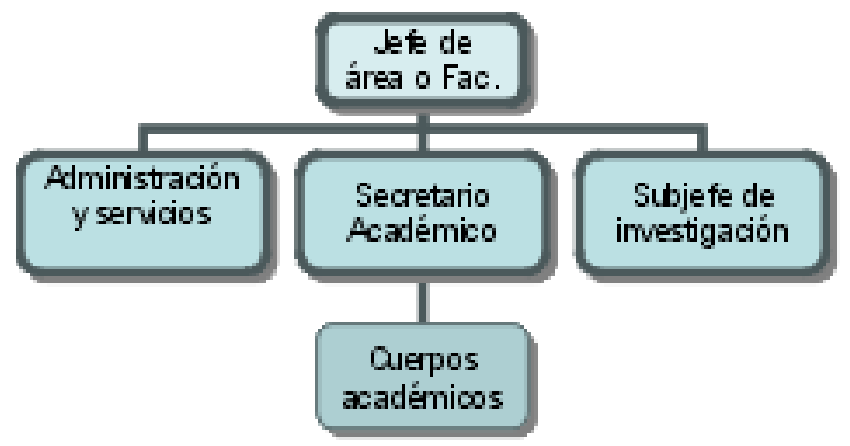

2. Convertir a los CA en círculos de calidad al estilo japonés sin facultades de decisión y acción (ver figura 5). Éste se trata de un modelo híbrido entre el burocrático y el colegiado. Es un modelo muy probable y no menos perjudicial, en muchos casos. 


\section{Figura 5}

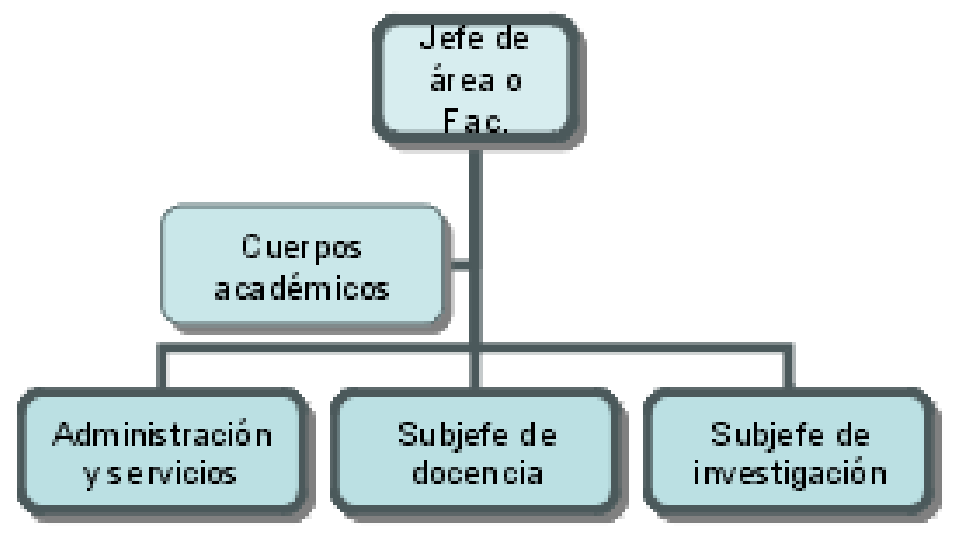

Finalmente, el denominado Programa de Fortalecimiento Institucional (PIFI), de la Secretaría de Educación Pública, es el medio adecuado para expresar y comprometer el plan de ajuste, siempre y cuando exista el compromiso de un cambio real por parte de los académicos y de las autoridades universitarias; en él se deberán expresar todas las adecuaciones necesarias de la normatividad, incluyendo la legitimación de la autoridad de los CA y su relación con el jefe de departamento. Ello requerirá una mayor claridad y concisión en la actuación de las autoridades, pues las inercias son muy grandes y la tentación por la simulación también.

Es necesario reconocer que todo cambio genera conflictos y desarreglo de intereses; así, el que se plantea (por su trascendencia y radicalidad) no es nada fácil de llevar a cabo. Sin embargo, si se pretende realizar una verdadera transformación en lo que hasta ahora ha sido la forma de operación de las universidades públicas en México, es necesario asumir los riesgos — por parte de las autoridades universitarias - y el compromiso de los actores - los académicos - para que dicha transformación, difícil y dolorosa para muchos, sea en verdad para beneficio de la universidad pública en México para que ésta no pierda el sentido social que debiera caracterizarla. 


\section{Bibliografía}

ANUIES, "La educación superior en el siglo XXI. Líneas estratégicas de desarrollo". (1999), en http://www.anuies.mx , diciembre 2001.

ARECHAVALA, R. y P. Solis, (1999). La universidad pública, ¿tiene rumbo su desarrollo? México: Universidad de Guadalajara-Universidad de Aguascalientes

BECHER, Tony. (2001). Tribus y territorios académicos. España: Gedisa.

BESUNSAN, G., E. de la Garza, F. Furstemberg, y C. Teran: Wannoffel, M. (1995). Competitividad internacional versus democracia industrial. México: UAMFundación F. Ebert.

CLARK, B. R. (1995). Places of inquiry; research and advanced education in modern universities. USA: University of California Press.

CLARK, B. R. (1983). El sistema de educación superior, una visión comparada de la organización académica. México: Nueva Imagen, UAM-A.

CONACYT, "Convocatoria de ciencia básica 2003-2", en http://www.conacyt.mx, diciembre 2003.

GIL, A. et al. (1994). Los rasgos de la diversidad, un estudio sobre los académicos mexicanos. UAM, México.

IBARRA, E. (2001). La universidad en México hoy: gubernamentalidad y modernización. México: UNAM-UAM-ANUIES.

MARCH, J., G. (1994). A primer on Decision Making. How decision happen. New York: Free Press.

MARCH, J. (1989). Decisions and Organizations. UK: Basil Blackwell.

MONTAÑO, L. (2001). "Los nuevos desafíos de la docencia. Hacia la construcción — siempre inacabada - de la Universidad". En A. Barba, L. Montaño, 
Universidad, organización y sociedad: arreglos y controversias (105132). México: Miguel Ángel Porrúa-UAM.

PÉREZ, Grediaga, Gil, Casillas, De Garay y Pizzonia (1991). "Los académicos de las universidades mexicanas. Contexto, discusión conceptual y dimensiones relevantes para la investigación". Sociológica 6 (15), UAM-Azcapotazalco, pp. 323-347.

PORTER, L. (2003). La universidad de papel, ensayos sobre la educación superior en México. México: CEIICH-UNAM.

PROMEP, Preguntas más frecuentes sobre CA's. En http://promep.sep.gob.mx/ ca.htm, diciembre 2003.

PROMEP, (1996). Programa de mejoramiento del profesorado. (SEP-ANUIESCONACYT).

RUBIO-OCA (2003), Documento presentado en la Universidad Autónoma de Yucatán. Mecanoescrito.

RUBIO-OCA, Julio. (1997). "Cuerpos Académicos". Omnia, 13(36), México, Anuies, pp. 15-19.

SEP (2003). Guia PIFI 3.0. Lineamientos para su actualización, formulación y presentación, México. SEP.

SOLÍS, P. (2001). "Universidad, Organización y Sociedad. Saberes Organizacionales: alcances y limitaciones de la certificación". En A. Barba, L. Montaño, Universidad, organización y sociedad: arreglos y controversias (133-161). México: Porrúa y UAM.

TAYLOR, P. G. (1999). Making Sense of Academic Life. Academics, Universities and change. Great Britain: The Society for Research into Higher Education \& Open University Pres.

WEICK, K. (1976) "Educational Organizations as loosely-coupled systems". Administrative Science Quaterly, 21: 1-19. C 